\title{
IMPROVED APPROACH FOR DESIGN OF PERFECT RECONSTRUCTION FIR QMF BANKS, WITH LOSSLESS LATTICE STRUCTURES
}

\author{
P. P. Vaidyanathan ${ }^{\frac{1}{4}}$, T. Q. Nguyen ${ }^{\frac{\hbar}{4}}$ and T. Saramaki* \\ †Dept. EE, Caltech, Pasadena, CA 91125 \\ ${ }^{*}$ Dept. EE, Tampere Univ. Tech., Finland
}

D3.9

\begin{abstract}
A new property of FIR lossless systems is introduced, leading to substantial improvement in the design procedure for perfect reconstruction QMF banks. The property enables the designer to initialize the coefficients of a lattice structure (which characterizes the analysis bank), in such a way as to speed up the convergence.
\end{abstract}

\section{Introduction}

Quadrature mirror filter banks (QMF) and their applications are well-known in the multirate digital signal processing community [1-3]. For a more complete reference list see [3]. A maximally decimated QMF bank (see Fig. 1, [4] ) is said to have the perfect-reconstruction (PR) property if the reconstructed signal $\hat{x}(n)$ is equal to a delayed (and possibly scaled) version of $x(n)$. A method was outlined in [4] for enforcing the PR property, based on the losslessness of the Alias-component (AC [5]) matrix. ${ }^{2}$ Let $H_{k}(z), 0 \leq k \leq M-1$ denote the $M$ analysis filters, and define the $M$ polyphase components of $H_{k}(z)$ to be $E_{k \ell}(z)$, $0 \leq \ell \leq M-1$ as in $[3,4]$. It is shown in [4] that a perfect reconstruction FIR QMF bank can be designed if the $M \times M$ matrix $\mathbf{E}(z) \triangleq\left[E_{k \ell}(z)\right]$ is lossless (this condition being equivalent to the losslessness of the $\mathrm{AC}$ matrix). With $\mathbf{E}(z)$ constrained to be lossless, the QMF bank has the PR property if and only if the synthesis filter coefficients $f_{k}(n)$ are time-reversed versions of $h_{k}(n)$.

In a subsequent work [6], a lattice structure has been presented (based on a state-space approach)in order to parametrize the complete family of $M \times M$ FIR lossless matrices $\mathbf{E}(z)$ with a given degree $N-1$ (the term degree stands for the McMillan degree [8], i.e., the minimum number of scalar delays $z^{-1}$ required for its realization). This parameterization has the following features: 1) the parameters are angles $\theta_{k} ; 2$ ) the number of parameters used is minimal, 3) regardless of the parameter values, the transfer matrix $\mathbf{E}(z)$ is guaranteed to be lossless, and 4) every $M \times M$ FIR lossless $\mathbf{E}(z)$ with a given degree is covered by the structure.

For FIR systems, losslessness of $\mathbf{E}(z)$ is equivalent to

Work supported in part by the National Science Foundation grants DCI 8552579 and MIP 8604456.

${ }^{2}$ Unless noted otherwise, the notations, terminology, and definitions used here are same as in our previous publications $[3,4]$. the property $\tilde{\mathbf{E}}(z) \mathbf{E}(z)=$ I. If $\mathbf{E}(z)$ has real coefficients, the lossless property becomes the LBR property [9]. For simplicity we shall restrict our discussions to this special case. In this case the number of angles $\theta_{k}$ required for parameterization is given by

$N_{p}=\left(\begin{array}{c}N-1+M \\ 2\end{array}\right)-\left(\begin{array}{c}N \\ 2\end{array}\right)=(M-1)(N-1)+\left(\begin{array}{c}M \\ 2\end{array}\right)$.

With the analysis filters so characterized in terms of $\mathbf{E}(z)$, the design problem is to optimize $\theta_{k}$ such that an objective function $\phi$ (sum of stopband energies of $H_{k}(z)[4]$ ) is minimized. Since $\phi$ is highly nonlinear, the optimization time becomes large as $M$ and $N$ increase. One aim of this paper is to show how this growth can be arrested to some extent, by using an important property of FIR lossless systems. The other purpose is to obtain an initialization for the optimization, based on $M$ th band filter theory, so as to further reduce the convergence-time.

Given an implementation of an $r$-input p-output system, we shall label the outputs of the delay elements as the state variables and obtain the description

$$
\begin{gathered}
\mathbf{x}(n+1)=\mathbf{A x}(n)+\mathbf{B u}(n) \\
\mathbf{y}(n)=\mathbf{C x}(n)+\operatorname{Du}(n) .
\end{gathered}
$$

The quadrupule $[\mathbf{A}, \mathbf{B}, \mathbf{C}, \mathbf{D}]$ is the 'state-space description' of the implementation.

\section{New results on the realization of FIR lossless systems}

In Sec. III, we shall design the FIR filters $H_{k}(z)$ as follows: first choose $H_{0}(z)$ to be an FIR $M$ th band filter [10] with 'good' stopband attenuation, and based on this $H_{0}(z)$, initialize 'as many parameters $\theta_{k}$ as possible' where $\theta_{k}$ are the parameters described earlier. The quantity of fundamental interest therefore is the number of freedoms that are left over, after constraining $H_{0}(z)$ to be a specific function. We shall study this issue here.

First, let $\mathrm{e}_{0}(z)$ be an $M \times 1$ FIR LBR vector, i.e.,

$$
\mathbf{e}_{0}(z)=\left[\begin{array}{llll}
e_{00}(z) & e_{01}(z) & \ldots & e_{0, M-1}(z)
\end{array}\right]^{T}
$$

with

$$
\tilde{\mathbf{e}}_{0}(z) \mathbf{e}_{0}(z)=1
$$


Then $\mathbf{e}_{0}(z)$ can be realized as in Fig. 1 , where $\hat{\mathbf{F}}(z)$ is $M \times M$ FIR LBR and $\hat{\mathbf{q}}_{0}$ is a real column vector with unit norm, i.e., $\hat{\mathrm{q}}_{0}^{T} \hat{\mathrm{q}}_{0}=1$. The synthesis procedure, which identifies the coefficients of $\hat{\mathbf{F}}(z)$ and the elements of $\hat{\mathbf{q}}_{0}$, can be found in [11]. As shown in [11], $\hat{\mathbf{F}}(z)$ and $\hat{\mathbf{q}}_{0}$ are not unique, for a given $\mathbf{e}_{0}(z)$. Fig. 1 says that we can write

$$
\mathbf{e}_{0}(z)=\hat{\mathbf{F}}(z) \hat{\mathbf{q}}_{0} \text {. }
$$

As a second step, let $\mathbf{e}_{k}(z), 0 \leq k \leq M-1$ be a set of FIR LBR vectors such that

$$
\tilde{\mathbf{e}}_{k}(z) \mathbf{e}_{l}(z)=\delta[k-l]
$$

where $\delta[k-l]$ is the Kronecker delta function. Then there exists a single $M \times M$ FIR LBR matrix $\mathbf{F}(z)$ such that all of the $\mathbf{e}_{k}(z)$ 's can be realized by using this $\mathbf{F}(z)$ as

$$
\mathbf{e}_{k}(z)=\mathbf{F}(z) \mathbf{q}_{k}
$$

where $\mathbf{q}_{k}$ are appropriate unit-norm real vectors such that

$$
\mathbf{q}_{k}^{T} \mathbf{q}_{l}=\delta[k-l] .
$$

This is a consequence of the results in [6],[7]. Here is a brief outline of the proof: define the $M \times M$ matrix

$$
\mathbf{S}(z)=\left[\begin{array}{llll}
\mathbf{e}_{0}(z) & \mathbf{e}_{1}(z) & \ldots & \mathbf{e}_{M-1}(z)
\end{array}\right] .
$$

Then S( $(z)$ is FIR LBR in view of (7). So it can be realized (see [6]) as in Fig. 2 where $\mathbf{F}(z)$ is an appropriately defined $M \times M$ FIR LBR matrix and $\mathrm{Q}$ is an appropriate real orthogonal matrix. In other words, we have

$$
\mathbf{S}(z)=\mathbf{F}(z) \mathbf{Q} \text {. }
$$

If we take $\mathbf{q}_{k}$ to be the $k$ th column of $\mathbf{Q}$, the above result (8) follows immediately. Once again, the matrices $\mathbf{F}(z)$ and $\mathbf{Q}$ are not unique for a given set of $\mathbf{e}_{k}(z), 0 \leq k \leq M-1$. We shall now prove the following main result.

Theorem 2.1. Let $\mathbf{e}_{k}(z), 0 \leq k \leq M-1$ be a set of $M \times 1$ FIR LBR vectors satisfying (7). Let $\mathbf{e}_{0}(z)$ be realized as in (6) using the procedure in [11]. Then all the vectors $\mathbf{e}_{k}(z)$ can be realized as $\mathbf{e}_{k}(z)=\hat{\mathbf{F}}(z) \hat{\mathbf{q}}_{k}$ where $\hat{\mathbf{q}}_{k}$ are appropriate unit-norm vectors.

Proof: Let us assume that the $M \times 1$ system $\mathbf{e}_{0}(z)$ has been realized as in (6) using the procedure in [11]. Assume that the $M$-input $M$-output core system $\hat{\mathbf{F}}(z)$ has state space description $\left[\hat{\mathbf{A}}, \hat{\mathbf{B}}_{c}, \hat{\mathbf{C}}, \hat{\mathbf{D}}_{c}\right]$, i.e.,

$$
\hat{\mathbf{F}}(z) \rightarrow\left[\hat{\mathbf{A}}, \hat{\mathbf{B}}_{c}, \hat{\mathbf{C}}, \hat{\mathbf{D}}_{c}\right] .
$$

The state-space description of $\mathbf{e}_{0}(z)$ is then $\left[\hat{\mathbf{A}}, \hat{\mathbf{B}}_{0}, \hat{\mathbf{C}}, \hat{\mathbf{D}}_{0}\right]$ where $\hat{\mathbf{B}}_{0}=\hat{\mathbf{B}}_{c} \hat{\mathbf{q}}_{0}$ and $\hat{\mathbf{D}}_{0}=\hat{\mathbf{D}}_{c} \hat{\mathbf{q}}_{0}$. Thus

$$
\mathbf{e}_{0}(z)=\hat{\mathbf{F}}(z) \hat{\mathbf{q}}_{0} \rightarrow\left[\hat{\mathbf{A}}, \hat{\mathbf{B}}_{c} \hat{\mathbf{q}}_{0}, \hat{\mathbf{C}}, \hat{\mathbf{D}}_{c} \hat{\mathbf{q}}_{0}\right] .
$$

Next, with $\mathbf{S}(z)$ defined as in (10), consider its realization as in Fig. 2. Let $\left[\mathbf{A}, \mathbf{B}_{c}, \mathbf{C}, \mathbf{D}_{c}\right\}$ be the state-space description of the core system $\mathbf{F}(z)$. Then the state space description of $\mathbf{S}(z)$ is given by $[\mathbf{A}, \mathbf{B}, \mathbf{C}, \mathbf{D}]$ where $\mathbf{B}=\mathbf{B}_{c} \mathbf{Q}$ and
$\mathbf{D}=\mathbf{D}_{c} \mathbf{Q}$. Now denoting the columns of $\mathbf{Q}$ by $\mathbf{q}_{k}$, we see that $\mathbf{e}_{k}(z)$ has the following relalization

$$
\mathbf{e}_{k}(z)=\mathbf{F}(z) \mathbf{q}_{k} \rightarrow\left[\mathbf{A}, \mathbf{B}_{c} \mathbf{q}_{k}, \mathbf{C}, \mathbf{D}_{c} \mathbf{q}_{k}\right] .
$$

In particular, $\mathbf{e}_{0}(z)$ has the realization

$$
\mathbf{e}_{0}(z)=\mathbf{F}(z) \mathbf{q}_{0} \rightarrow\left[\mathbf{A}, \mathbf{B}_{c} \mathbf{q}_{0}, \mathbf{C}, \mathbf{D}_{c} \mathbf{q}_{0}\right] .
$$

From the results of $[6]$ and $[7]$ we know that the two realizations (13) and (15) for $\mathbf{e}_{0}(z)$ are minimal (in the sense that the $\mathrm{A}$ matrix has the smallest size, say, $N-1 \times N-1$ ) and are related by a similarity transformation $[8]$ so that

$$
\left[\begin{array}{cc}
\hat{\mathbf{A}} & \hat{\mathbf{B}}_{0} \\
\hat{\mathbf{C}} & \hat{\mathbf{D}}_{0}
\end{array}\right]=\left[\begin{array}{cc}
\mathbf{T}^{-1} \mathbf{A} \mathbf{T} & \mathbf{T}^{-1} \mathbf{B}_{\mathrm{c}} \mathbf{q}_{0} \\
\mathbf{C T} & \mathbf{D}_{c} \mathbf{q}_{0}
\end{array}\right] \text {. }
$$

In summary, $\mathbf{e}_{0}(z)=\mathbf{F}(z) \mathbf{q}_{0}$, with $\mathbf{F}(z)$ realized as

$$
\mathbf{F}(z) \rightarrow\left[\hat{\mathbf{A}}, \mathbf{T}^{-1} \mathbf{B}_{c}, \hat{\mathbf{C}}, \mathbf{D}_{c}\right] .
$$

By the same argument, $\mathbf{e}_{k}(z)$ has a realization of the form $\mathbf{F}(z) \mathbf{q}_{k}$ with $\mathbf{F}(z)$ realized as in (17). Now consider the following $(N-1+M) \times(N-1+M)$ matrices

$$
\Theta=\left[\begin{array}{cc}
\hat{\mathbf{A}} & \hat{\mathbf{B}}_{c} \\
\hat{\mathbf{C}} & \hat{\mathbf{D}}_{c}
\end{array}\right], \quad \Sigma=\left[\begin{array}{cc}
\hat{\mathbf{A}} & \mathbf{T}^{-1} \mathbf{B}_{c} \\
\hat{\mathbf{C}} & \mathbf{D}_{c}
\end{array}\right] .
$$

It is known that $\Theta$ is orthogonal [6]. As a result we have

$$
\hat{\mathbf{A}}^{T} \hat{\mathbf{A}}+\hat{\mathbf{C}}^{T} \hat{\mathbf{C}}=\mathbf{I} \text {. }
$$

Combining this with the fact that $\Sigma$ represents a realization of the LBR matrix $\mathbf{F}(z)$, we conclude [12, page 919] that $\boldsymbol{\Sigma}$ is also orthogonal. As a result the columns of $\left[\begin{array}{l}\hat{\mathbf{B}}_{c} \\ \hat{\mathbf{D}}_{c}\end{array}\right]$ and $\left[\begin{array}{c}\mathbf{T}^{-1} \mathbf{B}_{c} \\ \mathbf{D}_{c}\end{array}\right]$ span the same subspace of rank $M$ (viz., the space orthogonal to the column-space of $\left[\begin{array}{l}\hat{\mathbf{A}} \\ \hat{\mathbf{C}}\end{array}\right]$. This implies the existence of an $M \times M$ matrix $\mathbf{R}$ such that

$$
\mathbf{T}^{-1} \mathbf{B}_{c}=\hat{\mathbf{B}}_{c} \mathbf{R}, \quad \mathbf{D}_{c}=\hat{\mathbf{D}}_{c} \mathbf{R} .
$$

Thus $\mathbf{F}(z)$ has the realization

$$
\mathbf{F}(z) \rightarrow\left[\hat{\mathbf{A}}, \hat{\mathbf{B}}_{c} \mathbf{R}, \hat{\mathbf{C}}, \hat{\mathbf{D}}_{c} \mathbf{R}\right] .
$$

Combining (12) and (21) we get the relation

$$
\mathbf{F}(z)=\hat{\mathbf{F}}(z) \mathbf{R} .
$$

Since $\mathbf{e}_{k}(z)$ has the realization (14), we conclude that it can also be realized as

$$
\mathbf{e}_{k}(z)=\hat{\mathbf{F}}(z) \hat{\mathbf{q}}_{k}, \quad \hat{\mathbf{q}}_{k}=\mathbf{R} \mathbf{q}_{k}
$$

In view of the fact that $\mathbf{e}_{k}(z)$ and $\hat{\mathbf{F}}(z)$ are both lossless, it can also be verified that $\hat{\mathbf{q}}_{k}$ has unit norm. This concludes the proof of the theorem.

We now have a simplified procedure for synthesiszing an $M \times M$ FIR LBR system $\mathrm{S}(z)$. The procedure is to synthesize only one column, say $\mathbf{e}_{0}(z)$, using the simple 
method in [11]. This gives a realization which can be characterized as in (6), where $\hat{\mathbf{F}}(z)$ is the cascaded lattice structure which results as a by-product [11] while synthesizing $\mathbf{e}_{0}(z)$. The remaining columns $\mathbf{e}_{k}(z)$ are synthesized simply by identifying unit-norm vectors

$$
\hat{\mathbf{q}}_{k} \triangleq \hat{\mathbf{F}}^{-1}(z) \mathbf{e}_{k}(z) \text {. }
$$

The inverse matrix on the RHS of (24) is equal to $\tilde{\hat{\mathbf{F}}}_{k}(z)$, see [9]. Even though the RHS of (24) appears to be a function of $z$ it is guaranteed to turn out to be a constant upon simplification. If we now define the $M \times M$ matrix $\hat{\mathbf{Q}} \triangleq\left[\begin{array}{llll}\hat{\mathbf{q}}_{0} & \hat{\mathbf{q}}_{1} & \ldots & \hat{\mathbf{q}}_{M-1}\end{array}\right]$ then $\mathbf{S}(z)=\hat{\mathbf{F}}(z) \hat{\mathbf{Q}}$, which can be readily implemented, since the structure realizing $\hat{\mathbf{F}}(z)$ is already available as the by-product mentioned above.

\section{New analysis-bank design procedure with lossless $\mathbf{E}(z)$}

We are interested in designing the $M$ FIR filters $H_{k}(z)$, $0 \leq k \leq M-1$ such that the matrix $\mathbf{E}(z)$ of polyphase components defined in Sec. I is LBR. For simplicity assume the coefficients of $H_{k}(z)$ to be real. One consequence [4] of requiring $\mathbf{E}(z)$ to be lossless is that the $\mathrm{AC}$ matrix $\mathbf{H}(z)$ is lossless. Each $H_{k}(z)$ in particular satisfies

$$
\sum_{l=0}^{M-1} H_{k}\left(z W^{l}\right) H_{k}\left(z^{-1} W^{-l}\right)=c
$$

where $c$ is a constant. In other words, $G_{k}(z) \triangleq H_{k}\left(z^{-1}\right) H_{k}(z)$ is an $M$ th band (or Nyquist) linear-phase FIR filter [10] with nonnegative real frequency response $G_{k}\left(e^{j \omega}\right)$.

Suppose we have designed a low pass FIR $M$ th band filter $G_{0}(z)$ with $G_{0}\left(e^{j \omega}\right) \geq 0$. Let $H_{0}(z)$ be an arbitrary spectral factor of $G_{0}(z)$. Let the polyphase components of $H_{0}(z)$ be denoted by $E_{0 l}(z), 0 \leq l \leq M-1$. The property (25) ensures[4] that the vector

$$
\mathbf{e}_{0}(z) \triangleq\left[E_{00}(z) E_{01}(z) . . E_{0, M-1}(z)\right]^{T}
$$

is FIR LBR. Assume that this vector has been realized in the form (6) using the procedure in [11]. The vector $\mathbf{e}_{0}^{T}(z)$ is the first row of the polyphase component matrix $\mathbf{E}(z)$. We are now left with the task of finding the remaining $M-1$ rows $\mathbf{e}_{k}^{T}(z), k>0$ of $\mathbf{E}(z)$ such that $\mathbf{E}(z)$ is lossless and such that $H_{k}(z), k>0$ are 'good' bandpass filters with desired specifications. Recall that losslessness of $\mathbf{E}(z)$ is equivalent to the condition (7). But we know from Theorem 2.1 that if $(7)$ holds, and if $\mathbf{e}_{0}(z)$ has been realized as in (6), then all of $\mathbf{e}_{k}(z)$ can be realized as in (23). In other words, we have

$$
\mathbf{E}^{T}(z)=\hat{\mathbf{F}}(z) \hat{\mathbf{Q}}
$$

where $\hat{\mathbf{F}}(z)$ and the 0 th column $\hat{\mathbf{q}}_{0}$ of $\hat{\mathbf{Q}}$ are already fixed by the choice of $H_{0}(z)$. The the number of freedoms that remain for the design of $H_{k}(z), 1 \leq k \leq M-1$ (under the constraint that $\mathbf{E}(z)$ be LBR) is then equal to the number of freedoms we can exercise in choosing the columns $\hat{\mathbf{q}}_{k}$,
$1 \leq k \leq M-1$. Since $\mathbf{E}(z)$ and $\hat{\mathbf{F}}(z)$ are LBR, $\hat{\mathbf{Q}}$ is of course $M \times M$ orthogonal. So $\hat{\mathbf{Q}}$ has $\left(\begin{array}{c}M \\ 2\end{array}\right)$ freedoms [6]. The first column $\hat{\mathbf{q}}_{0}$ which is a unit norm vector has $M-1$ freedoms, which are already used up by the choice of $H_{0}(z)$. We are therefore left with

$$
N_{f}=\left(\begin{array}{c}
M \\
2
\end{array}\right)-(M-1)
$$

freedoms. Summarizing, we have

Lemma 3.1. Let $H_{0}(z)$ be a given FIR transfer function whose $M \times 1$ polyphase-component vector $\mathbf{e}_{0}(z)$ is LBR. Then the number of design freedoms available in choosing a set of $M-1$ transfer functions $H_{k}(z), 1 \leq k \leq M-1$ under the constraint that the complete $M \times M$ polyphase matrix $\mathbf{E}(z)$ of $H_{k}(z), 0 \leq k \leq M-1$ is LBR, is given by (27) regardless of the filter-lengths.

Consider, for example, the special case of $M=2$. As shown in [4], the matrix $\mathbf{E}(z)$ for the designs in [13] turns out to be lossless. Since $N_{f}=0$ in this case, we see that once $H_{0}(z)$ is chosen to be a spectral factor of a zero-phase half-band filter, there is no freedom in the choice of $H_{1}(z)$ ! This is completely compatible with the fact that $H_{1}(z)$ in [13] is constrained to be equal to $z^{-n_{0}} H_{0}\left(-z^{-1}\right)$.

Perhaps the most important part of the Lemma is the phrase 'regardless of the filter length'. As a consequence of this, we can optimize the frequency responses of $H_{k}(z), 1 \leq k \leq M-1$ (for a given $H_{0}(z)$ ) by varying only $N_{f}$ parameters which is much smaller than $N_{p}$ in (1).

A design procedure based on the above results would typically run as follows: first design a linear-phase FIR $M$ th band filter $G_{0}(z)$ with nonnegative response $G_{0}\left(e^{j \omega}\right)$ using, for example, the technique in [10]. Then identify a spectral factor of $G_{0}(z)$, and label it as one of the analysis filters, say $H_{0}(z) .{ }^{3}$ The choice of the length of $G_{0}(z)$ of course depends on the attenuation requirement of $H_{0}(z)$. Next, figure out the polyphase-component vector $\mathbf{e}_{0}(z)$. This is guaranteed to be LBR. Synthesize it using the technique in [11]. The result is in the form (6) where $\hat{\mathbf{q}}_{0}$ is a unit-norm vector and $\hat{\mathbf{F}}(z)$ is characterized[11] by $(N-1)(M-1)$ planar rotation angles $\theta_{k}$, where $N-1$ is the order of the highest-degree polyphase component. At this point, $\hat{\mathbf{F}}(z)$ and $\hat{\mathbf{q}}_{0}$ in (6) are completely specified. Optimize the $N_{f}$ remaining degrees of freedom to minimize the stopband energies of $H_{k}(z), 1 \leq k \leq M-1$.

A second alternative for the design procedure would be to optimize all the $N_{p}$ freedoms (see (1)), so that all the filters (including $H_{0}(z)$ ) tend to come out to be equally good. This of course takes longer computer-time, but is still much faster than the 'random initialization' used in [4].

A design example based on the method described above is shown in Fig. 3. Here $M=3$, and the analysis filters have order 55. The filter $H_{1}(z)$ (rather than $H_{0}(z)$ )

\footnotetext{
${ }^{3}$ It is possible to avoid general spectral factorization by use of a modified design of $G_{0}(z)$ based on the eigenfilters approach [15]. See $[16]$ for details.
} 
was obtained as a spectral factor of a 3rd band linearphase filter of order 110. Explicit spectral factorization was avoided by using the multistage eigenfilter method reported in [16], for the design of the 3rd band filter. The second alternative described in the preceding paragraph was then used to optimize all the three analysis filter responses. Fig. 3 shows the final optimized responses. Compared to earlier methods [4],[6], the new method converges much faster, and always leads to much improved attenuation characteristics for a given filter length.

\section{References:}

[1] R. E. Crochiere and L. R. Rabiner, Multirate digital signal processing, Prentice Hall, Inc., 1983.

[2] A. Croisier, D. Esteban, and C. Galand, "Perfect channel splitting by use of interpolation/decimation/ tree decomposition techniques," Int. Symp. on Info., Circuits and Systems, Patras, Greece, 1976.

[3] P.P. Vaidyanathan, "Quadrature mirror filter banks, $M$-band extensions and perfect-reconstruction techniques," IEEE ASSP magazine, pp. 4-20, July 1987.

[4] P.P. Vaidyanathan, "Theory and design of $M$-channel maximally decimated quadrature mirror filters with arbitrary $M$, having perfect reconstruction property," IEEE Trans. ASSP, pp. 476-492, April 1987.

[5] M. J. T. Smith and T. P. Barnwell III, "A unifying framework for analysis/synthesis systems based on maximally decimated filter banks," Proc. IEEE ICASSP, pp. 521-524, Tampa, FL, Mar. 1985.

[6] P. P. Vaidyanathan, Zinnur Doganata and Truong Q. Nguyen, "More results on the perfect reconstruction problem in $M$-band parallel QMF banks," Proc. IEEE ISCAS, pp. 847-850, Philadelphia, May 1987.

[7] P. P. Vaidyanathan, Zinnur Doganata and Truong Q. Nguyen, "General synthesis procedures for FIR lossless transfer matrices for perfect-reconstruction multirate filter bank applications," Tech. Rep. Caltech, Aug 1987.

[8] T. Kailath, Linear Systems, Prentice Hall, 1980.

[9] P. P. Vaidyanathan and S. K. Mitra, "Low passband sensitivity digital filters: A generalized viewpoint and synthesis procedures," Proc. IEEE, pp. 404-423, April 1984.

[10] F. Mintzer, "On half-band, third-band and $N$ th band FIR filters and their design," IEEE Trans. ASSP, pp.734-738, Oct. 1982.

[11] P. P. Vaidyanathan, "Passive cascaded lattice structures for low sensitivity FIR filter design, with applications to filter banks," IEEE Trans. CAS, pp. 1045-1064, Nov. 1986.

[12] P. P. Vaidyanathan, "The discrete-time bounded real lemma in digital filtering," IEEE Trans. CAS, pp. 918-924, Sept. 1985.
[13] M. J. T. Smith and T. P. Barnwell III, "Exact reconstruction techniques for tree-structured subband coders," IEEE Trans. on ASSP, pp. 434-441, June 1986.

[14] Truong Q. Nguyen and P. P. Vaidyanathan, "New lattice structures for the design of perfect reconstruction QMF banks with image-symmetry property," IASTED ISSPA Proc., Brisbane, Australia, Aug. 1987.

[15] P. P. Vaidyanathan and T. Q. Nguyen, "Eigenfilters: a new approach to least squares FIR filter design and applications including Nyquist filters," IEEE Trans. CAS, pp. 11-23, Jan 1987.

[16] T. Q. Nguyen, T. Saramaki and P. P. Vaidyanathan, "Eigenfilters for the design of special transfer functions with applications in multirate signal processing,” Proc. ICASSP 88, New York, NY, Apr. 1988.

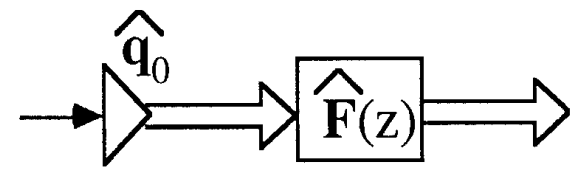

Fig. 1. Realization of $\mathbf{e}_{0}(\mathrm{z})$

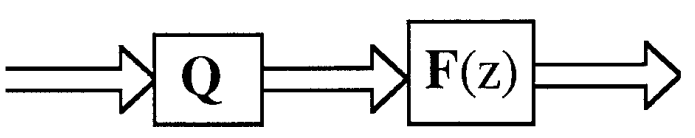

Fig. 2. Realization of $\mathbf{S}(\mathrm{z})$

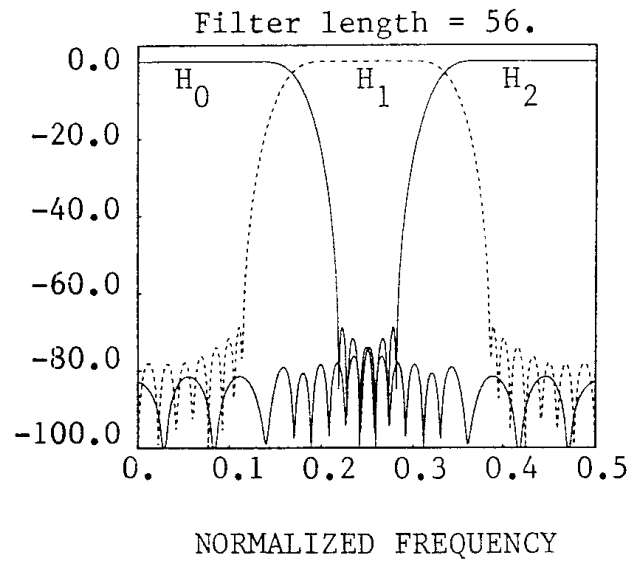

Fig. 3. Magnitude response in DB for optimized analysis filters. 